\title{
Associations between Intensive Lipid-lowering Therapy and Outcomes of Intracerebral Hemorrhage
}

\author{
Tianping Tang ${ }^{1}$, Ligong Zhang ${ }^{1}$, Xingquan $\mathrm{Zhao}^{2}$, Shimeng Liu ${ }^{2 *}$ \\ ${ }^{1}$ Department of Neurology, Shengli Oilfiled Central Hospital, 31 Jinan Road, Dongying, Shandong, China. 257000. \\ ${ }^{2}$ Department of Neurology, Beijing Tiantan Hospital, Capital Medical University, 110 W. South 4th Ring Road, Fengtai, Beijing, China. 100070. \\ *Corresponding Author: Shimeng Liu, Department of Neurology, Beijing Tiantan Hospital, Capital Medical University, 110 W. South 4th \\ Ring Road, Fengtai, Beijing, China. 100070. \\ Received date: July 22, 2021; Accepted date: July 29, 2021; Published date: August 05,2021 \\ Citation: Tianping Tang., Ligong Zhang., Xingquan Zhao., Shimeng Liu. (2021) Associations between Intensive Lipid-lowering Therapy and \\ Outcomes of Intracerebral Hemorrhage. J. Neuroscience and Neurological Surgery. 9(3); DOI:10.31579/2578-8868/200
}

Copyrights: () 2021 Shimeng Liu, This is an open-access article distributed under the terms of The Creative Commons Attribution License, which permits unrestricted use, distribution, and reproduction in any medium, provided the original author and source are credited

\begin{abstract}
:
Background: The intensive lipid-lowering therapy (ILLT), targeting an low-density lipoprotein cholesterol (LDL-C) $<1.80 \mathrm{mmol} / \mathrm{L}$, was a crucial strategy for the secondary prevention of cerebrovascular diseases. But the associations between ILLT and the outcomes after intracerebral hemorrhage (ICH) were unclear.

Materials and Methods: Data of the consecutive patients with acute ICH and past medical histories of ischemic stroke from 2017 to 2019 at an academic stroke center in China were analyzed. The study patients were classified according to their baseline LDL-C levels: $<1.80 \mathrm{mmol} / \mathrm{L}$ vs. $\geq 1.80 \mathrm{mmol} / \mathrm{L}$. The outcomes of ICH were compared between the two groups. Multivariate linear mixed effect model with repeated measures adjusting for ICH scores were used to determine the associations between LDL-C levels and the change in NIHSS scores; baseline ICH scores were adjusted in the multi-variable models

Results: A total of 197 patients were included in the study, 31 of them had LDL-C $<1.80 \mathrm{mmol} / \mathrm{L}$ and 166 had LDL$\mathrm{C} \geq 1.80 \mathrm{mmol} / \mathrm{L}$. We did not test any significant differences regarding the demographic characteristics or vascular risk factors. Medians of the baseline National Institutes of Health Stroke Scale (NIHSS) scores (8 vs. 9, P = 0.79) and ICH scores ( 1 vs.1, $\mathrm{P}=0.26$ ) were similar. But the patients with $\mathrm{LDL}-\mathrm{C}<1.80 \mathrm{mmol} / \mathrm{L}$ had higher risks of secondary intraventricular hemorrhage $(13 \%$ vs. $4 \%, \mathrm{P}=0.03)$. Outcomes of the hemorrhagic stroke at discharge were similar, except the patients with LDL-C $\geq 1.80 \mathrm{mmol} / \mathrm{L}$ had significant improvements in their NIHSS scores at discharge (estimated change in means: $-2.4,95 \% \mathrm{CI}:[-4.3,-0.5]$ ), while patients with LDL-C $<1.80 \mathrm{mmol} / \mathrm{L}$ did not (estimated change in means: $-1.4,95 \%$ CI: $[-5.9,3.0])$.

Conclusion: ILLT achieved LDL-C $<1.80 \mathrm{mmol} / \mathrm{L}$ was associated with limited improvements in the neurological deficits in the patients with ICH.

Keywords: intensive lipid lowering therapy; intracerebral hemorrhage; low-density cholesterol lipoprotein; outcomes
\end{abstract}

\section{Introduction}

Serum low-density lipoprotein cholesterol (LDL-C) was identified as an independent risk factor of ischemic stroke.[1] The use of the 3-hydroxy3-methylglutaryl coenzyme A reductase inhibitor (statin) class of drugs, reduces serum LDL-C by $55 \%$ to $60 \%$ at the maximal doses. Our prior study found achieving $\mathrm{LDL}-\mathrm{C}<1.80 \mathrm{mmol} / \mathrm{L}$ in ischemic stroke patients was associated with a trend of reducing atherosclerotic plaque progression at one year, with (1.4 mm decreased in atherosclerotic plaque length and $0.2 \mathrm{~mm}$ decreased in thickness. [2] Intensive lipid-lowering therapy (ILLT) with statin was recommended to reduce the risks of ischemic 
stroke.[1] But the impacts of ILLT on the intracerebral hemorrhage (ICH) was not well studied.

Spontaneous, nontraumatic ICH is a significant cause of morbidity and mortality, ranking the second subtype of stroke.[3, 4] The mortality rate of ICH was reported to be $40 \%$ at 1 month and $54 \%$ at 1 year, only 12 $39 \%$ of the survivors achieved long-term functional independence.[4] Risk factors of ICH included advanced age, hypertension, current smoking, excessive alcohol consumption, hypocholesterolemia.[4] Blood pressure managements, intensive care, glucose managements, surgery intervention was the standard managements of ICH.[3]

When balancing the benefits of ILLT for the prevention of ischemic stroke and the increased risks of $\mathrm{ICH}$, the current evidence was controversial. In a cumulative meta-analysis of lipid-lowering trials reported the events of $\mathrm{ICH}, 9.17$ (95\% confidence interval [CI]:[5.78, 12.66]) fewer ischemic strokes and 0.41 (95\% CI: [0.05, 0.86] more ICH per 1000 person-years were estimated.[5] Based on the French population cohort in the Treat Stroke to Target trial $(n=1073)$, after an ischemic stroke of documented atherosclerotic origin, lipid-lowering therapy targeting an LDL-C $<1.80 \mathrm{mmol} / \mathrm{L}$ during 5.3 years did not change the risks of $\mathrm{ICH}(1.2 \%$ vs. $1.0 \%$, Odds Ratio [OR]: $1.17 ; 95 \%$ CI: [0.53, 2.62]; $\mathrm{P}=0.70)$.[6] But according to the large $(\mathrm{n}=96,043)$ community based multi-center prospective cohort study in China, participants with LDL-C concentrations $<1.80 \mathrm{mmol} / \mathrm{L}$ had significantly higher risks of developing ICH than those with LDL-C concentrations within 1.80 - 2.56 mmol/L: adjusted hazard ratios (HR) was 1.65 (95\% CI: [1.32, 2.05]) and 2.69 (95\% CI: [2.03, 3.57]) for the patients with LDL-C concentrations within $1.30-1.79 \mathrm{mmol} / \mathrm{L}$ and $<1.30 \mathrm{mmol} / \mathrm{L}$, respectively.[7]

Evidence from the studies to investigate the associations between the serum LDL-C concentrations and the prognosis of ICH was even less. In a retrospective study of the patients with acute ICH $(n=672)$, higher admission LDL-C concentrations were independently associated with decreased risks of hematoma expansion (OR: 0.88, 95\% CI: [0.77, 0.99]; $\mathrm{P}=0.048$ ) and lower likelihood of in-hospital death (OR increased by 0.68 per $0.3 \mathrm{mmol} / \mathrm{L}$ decreased in LDL-C, $95 \% \mathrm{CI}$ : [0.57, 0.80]; P < 0.001).[8] The results were consistent with another small cohort study, where 136 patients with spontaneous ICH were prospectively evaluated, LDL-C levels were found to be significantly lower in the patients with hematoma expansion (3.19 vs. $3.70 \mathrm{mmol} / \mathrm{L} ; \mathrm{P}=0.003)$.[9] Whereas, in 572 patients with ICH selected from 2 centers of the cerebral small vessel disease cohort, LDL-C levels were not associated with the risks of hematoma expansion (OR: 1.11, 95\% CI: [0.75, 1.67]; P = 0.594).[10] Due the current limited studies, we carried the retrospective cohort study to explore the associations between the ILLT in the secondary stroke prevention settings and the outcomes of ICH.

\section{Materials and Methods:}

We retrospectively analyzed the data of the consecutive patients with acute ICH at a comprehensive stroke center in China from 2017 to 2019. The inclusion criteria were: $(1) \geq 18$ years old; (2) with the admission diagnosis of spontaneous ICH; (3) symptoms onset within 7 days; (4) with the past medical histories of ischemic stroke.[11] We excluded the patients with the etiology of vascular malformations.[11] Diagnosis and stroke subtypes were determined based on clinical features, brain imaging and angiography.[11] The study was approved by the institutional review board (IRB).

The study patients were stratified according to their baseline serum LDLC concentrations: $<1.80$ vs. $\geq 1.80 \mathrm{mmol} / \mathrm{L}$.[12] LDL-C $\geq 1.80 \mathrm{mmol} / \mathrm{L}$ was used as the reference level; as so to test the associations of ILLT (baseline LDL-C $<1.80 \mathrm{mmol} / \mathrm{L}$ ) and the outcomes of ICH.

The scores of National Institute of Health Stroke Scale (NIHSS) and Glasgow Coma Scale (GCS) were recorded in the emergent room to evaluate the baseline severity of the indexed events. [11] ICH scale was used to assess the severity of the ICH events. The modified Rankin Scales (mRS) were tested at discharge to evaluate the prognosis of the hemorrhagic events. An mRS score > 3 was defined as unfavorable outcomes. The ICH scale was ranged from 0 to 6 , with higher scores indicating higher risks of ICH.[13]

Data analysis first compared distributions of sex, age, vascular risk factors, medication history and results of blood work between the two groups (LDL-C: $<1.80$ vs. $\geq 1.80 \mathrm{mmol} / \mathrm{L}$ ). We used $\chi 2$ test or Fisher's exact tests to examine the distributions of each categorical variable and Kruskal-Wallis tests to examine the distributions of the non-normally distributed numerical variables, with a significance level of $\mathrm{P}<0.05$. Logistic regression models were used to compare the categorical outcomes.

Multivariate linear mixed effect models with repeated measures adjusting for ICH scores were used to determine the associations between LDL-C levels and the change in NIHSS scores; baseline ICH scores were adjusted in the multi-variable models

The SAS statistical package (version 9.4; SAS Institute, Cary, NC, USA) was used to perform data analysis.

\section{Results}

\section{Study patients}

A total of 197 patients were included in the study. They were 131 men and 68 women. The median age of the study patients were 65 years. In the entire study cohort, $180(91 \%)$ patients had mRS scores less than 3 ahead of the hemorrhagic events, and $161(82 \%)$ had been taking statin (Table 1). 
Table 1: Baseline characteristics of study patients

\begin{tabular}{|c|c|c|c|c|c|}
\hline & $\begin{array}{l}\text { LDL-C } \\
<1.80 \mathrm{mmol} / \mathrm{L} \\
\quad(\mathrm{n}=\mathbf{3 1})\end{array}$ & $\begin{array}{c}\text { LDL-C } \\
\geq 1.80 \\
\mathrm{mmol} / \mathrm{L} \\
(\mathrm{n}=166)\end{array}$ & $\begin{array}{l}\text { All patients } \\
\quad(n=197)\end{array}$ & Statistics & $P$-value \\
\hline \multicolumn{6}{|l|}{ Demographic characteristics } \\
\hline Age, y, median (IQR) & $62(51-70)$ & $67(54-73)$ & $65(53-73)$ & $1.94^{*}$ & 0.16 \\
\hline Male, $(\mathrm{n}, \%)$ & $24(77 \%)$ & $107(64 \%)$ & $131(67 \%)$ & $1.97^{\dagger}$ & 0.16 \\
\hline \multicolumn{6}{|l|}{ Medical histories } \\
\hline Pre-mRS scores, median (IQR) & $0(0-1)$ & $0(0-1)$ & $0(0-1)$ & $0.12^{*}$ & 0.73 \\
\hline Pre-mRS scores $0-2,(\mathrm{n}, \%)$ & $28(90 \%)$ & $152(92 \%)$ & $180(91 \%)$ & $0.74^{\dagger}$ & 0.25 \\
\hline Cigarette smoking, n (\%) & $16(52 \%)$ & $82(49 \%)$ & $98(50 \%)$ & $0.05^{\dagger}$ & 0.82 \\
\hline Alcohol consuming, $\mathrm{n}(\%)$ & $10(32 \%)$ & $35(21 \%)$ & $45(23 \%)$ & $1.85^{\dagger}$ & 0.17 \\
\hline Atrial Fibrillation, $\mathrm{n}(\%)$ & $0(0 \%)$ & $1(1 \%)$ & $1(1 \%)$ & $0.84 *$ & 1.0 \\
\hline Coronal Heart Disease, $\mathrm{n}(\%)$ & $5(16 \%)$ & $30(18 \%)$ & $35(18 \%)$ & $0.07^{\dagger}$ & 0.80 \\
\hline Diabetes, n (\%) & $3(10 \%)$ & $12(7 \%)$ & $15(8 \%)$ & $0.24^{*}$ & 0.71 \\
\hline Hypertension, n (\%) & $18(58 \%)$ & $72(44 \%)$ & $90(46 \%)$ & $2.27^{\dagger}$ & 0.13 \\
\hline Statin use, $\mathrm{n}(\%)$ & $24(77 \%)$ & $137(83 \%)$ & $161(82 \%)$ & $0.46^{\dagger}$ & 0.50 \\
\hline Antiplatelets, n (\%) & $26(84 \%)$ & $146(88 \%)$ & $172(87 \%)$ & $0.39^{\dagger}$ & 0.53 \\
\hline Anticoagulation, $\mathrm{n}(\%)$ & $0(0 \%)$ & $4(2 \%)$ & $4(2 \%)$ & $0.50^{*}$ & 1.0 \\
\hline Hypertension medication, $\mathrm{n}(\%)$ & $24(77 \%)$ & $137(83 \%)$ & $161(82 \%)$ & $0.46^{\dagger}$ & 0.50 \\
\hline \multicolumn{6}{|l|}{ Blood Tests } \\
\hline HbA1c, \%, median (IQR) & $5.6(5.0-6.5)$ & $5.9(5.0-7.0)$ & $5.8(5.0-6.8)$ & $0.79^{*}$ & 0.38 \\
\hline Glucose, mmol/L, median (IQR) & $5.8(4.6-8.2)$ & $5.7(4.6-7.5)$ & $5.7(4.6-7.5)$ & $0.28^{*}$ & 0.59 \\
\hline $\mathrm{TG}, \mathrm{mmol} / \mathrm{L}$, median (IQR) & $0.76(0.48-1.12)$ & $1.02(0.70-1.39)$ & $0.94(0.67-1.38)$ & $1.00^{*}$ & 0.32 \\
\hline Tch, mmol/L, median (IQR) & $2.89(2.67-3.50)$ & $4.34(3.67-4.82)$ & $4.14(3.45-4.75)$ & $64.59^{*}$ & $<0.0001$ \\
\hline HDL-C, mmol/L, median (IQR) & $1.11(0.92-1.55)$ & $1.17(1.00-1.38)$ & $1.17(1.00-1.43)$ & $0.03^{*}$ & 0.85 \\
\hline LDL-C, mmol/L, median (IQR) & $1.49(1.25-1.77)$ & $2.70(2.27-3.17)$ & $2.56(2.04-3.08)$ & $77.98^{*}$ & $<0.0001$ \\
\hline
\end{tabular}

*Kruskal-Wallis test; $†$ Chi-Square test $;$ fFisher's Exact test.

Abbreviations: IQR: interquartile range; $\boldsymbol{m R S}$ : modified Rankin Scale; HbAlc: glycated hemoglobin; TG: triglyceride; Tch: total cholesterol; HDLC: high-density lipoprotein cholesterol; LDL-C: low-density lipoprotein cholestero

Thirty-one patients had serum LDL-C $<1.80 \mathrm{mmol} / \mathrm{L}$ and 166 patients had serum LDL-C $\geq 1.80 \mathrm{mmol} / \mathrm{L}$ at admission. The median of serum LDL-C was $1.49 \mathrm{mmol} / \mathrm{L}$ and $2.70 \mathrm{mmol} / \mathrm{L}$ in the patients with $\mathrm{LDL}-\mathrm{C}<$ $1.80 \mathrm{mmol} / \mathrm{L}$ and $\geq 1.80 \mathrm{mmol} / \mathrm{L}$, respectively $(\mathrm{P}<0.0001)$. The distributions of previous statin use did not vary between the two groups (LDL-C $<1.80 \mathrm{mmol} / \mathrm{L}$ vs. $\geq 1.80 \mathrm{mmol} / \mathrm{L}: 77 \%$ vs. $83 \%$; $\mathrm{P}=0.50$ ). We did not test any significant differences regarding the demographic characteristics or the vascular risk factors (Table 1).

The median of the baseline NIHSS scores in the entire study population was 9. We did not find any differences in the baseline NIHSS scores between the two groups (LDL-C $<1.80 \mathrm{mmol} / \mathrm{L}$ vs. $\geq 1.80 \mathrm{mmol} / \mathrm{L}: 8 \mathrm{vs}$.
9; $\mathrm{P}=0.79)$. The median ICH score was 1 in either group $(\mathrm{P}=0.26)$. The rate of secondary intraventricular hemorrhage was $13 \%$ in patients with $\mathrm{LDL}-\mathrm{C}<1.80 \mathrm{mmol} / \mathrm{L}$, and $4 \%$ in patients with $\mathrm{LDL}-\mathrm{C} \geq 1.80 \mathrm{mmol} / \mathrm{L}$ $(\mathrm{P}=0.03)$. In the logistic model, patients with $\mathrm{LDL}-\mathrm{C} \geq 1.80 \mathrm{mmol} / \mathrm{L}$ has $10 \%$ lower risks of having intraventricular bleeding (OR: $0.92,95 \% \mathrm{CI}$ : $[0.46,1.83], \mathrm{P}=0.81)$. Deep locations were the most frequently $(67 \%)$ affected sites, but no differences were shown between the two groups (Table 2). Herniation happened in $12 \%$ patients (LDL-C $<1.80 \mathrm{mmol} / \mathrm{L}$ vs. $\geq 1.80 \mathrm{mmol} / \mathrm{L}: 19 \%$ vs. $10 \% ; \mathrm{P}=0.15)$. In the logistic model, patients with $\mathrm{LDL}-\mathrm{C} \geq 1.80 \mathrm{mmol} / \mathrm{L}$ was less likely to have herniation (OR: 0.48, 95\% CI: [0.23, 0.98], $\mathrm{P}=0.04)$.

Table 2: Characteristics of the indexed intracerebral hemorrhagic events

\begin{tabular}{|c|c|c|c|c|c|}
\hline & $\begin{array}{c}\text { LDL-C } \\
<1.80 \mathrm{mmol} / \mathrm{L} \\
(\mathrm{n}=31)\end{array}$ & $\begin{array}{c}\text { LDL-C } \\
\geq 1.80 \mathrm{mmol} / \mathrm{L} \\
(\mathrm{n}=166)\end{array}$ & $\begin{array}{c}\text { All } \\
\text { patients } \\
(\mathbf{n}=197)\end{array}$ & Statistics & $\boldsymbol{P}$ \\
\hline Baseline NIHSS scores, median, IQR & $8(3-23)$ & $9(4-17)$ & $9(4-17)$ & $0.07^{*}$ & 0.79 \\
\hline Baseline GSC scores, median, IQR & $12(5-15)$ & $13(9-15)$ & $13(8-15)$ & $1.27^{*}$ & 0.26 \\
\hline Baseline GSC score < 15, n (\%) & $21(68 \%)$ & $99(60 \%)$ & $120(61 \%)$ & $0.72^{\dagger}$ & 0.4 \\
\hline ICH scores"II, median, IQR & $1(0-3)$ & $1(0-2)$ & $1(0-2)$ & 0.31 & 0.26 \\
\hline Bleeding Volume, ml, median (IQR) & $10(6-58)$ & $11(4-22)$ & $10(4-24)$ & $1.46^{*}$ & 0.23 \\
\hline Primary Bleeding locations, $\mathrm{n}(\%)$ & & & & & \\
\hline
\end{tabular}




\begin{tabular}{|c|c|c|c|c|c|}
\hline Deep areas & $20(65 \%)$ & $112(67 \%)$ & $132(67 \%)$ & $2.41^{*}$ & 0.66 \\
\hline Lobar & $6(19 \%)$ & $33(20 \%)$ & $39(20 \%)$ & & \\
\hline Brainstems & $2(6 \%)$ & $10(6 \%)$ & $12(6 \%)$ & & \\
\hline Cerebellums & $1(3 \%)$ & $8(5 \%)$ & $9(5 \%)$ & & \\
\hline Intraventricular bleeding & $2(6 \%)$ & $3(2 \%)$ & $5(3 \%)$ & & \\
\hline $\begin{array}{l}\text { Secondary intraventricular } \\
\text { hemorrhage, } \mathrm{n}(\%)\end{array}$ & $4(13 \%)$ & $6(4 \%)$ & $10(5 \%)$ & $4.68^{*}$ & $0.03 *$ \\
\hline Herniation, n $(\%)$ & $6(19 \%)$ & $17(10 \%)$ & $23(12 \%)$ & $2.10^{\dagger}$ & 0.15 \\
\hline
\end{tabular}

$*$ Kruskal-Wallis test; $\dagger$ Chi-Square test; $\neq$ Fisher's Exact test; $\mathbf{9 I C H}$ score : a scale assessing the severity of ICH ranged from 0 to $6 . * P$-value $<0.05$. Abbreviations: NIHSS: National Institutes of Health Stroke Scale; ICH: intracranial hemorrhage. IQR: interquartile range; GCS: Glasgow Coma Scale; LDL-C: low-density lipoprotein cholesterol.

Patients with LDL-C $\geq 1.80 \mathrm{mmol} / \mathrm{L}$ have significant improvements in their NIHSS scores at discharge than baseline (estimated change in means: $-2.4,95 \%$ CI: $[-4.7,-0.1])$, but the patients with LDL-C $<1.80$ $\mathrm{mmol} / \mathrm{L}$ did not reach significant levels (estimated change in means: -1.4 , 95\% CI: $[-6.9,4.0])$. After adjusting baseline ICH scores, patients with LDL-C $\geq 1.80 \mathrm{mmol} / \mathrm{L}$ have significant improvements in their NIHSS scores at discharge (estimated change in means: $-2.4,95 \%$ CI: [-4.3, $0.5]$ ), while patients with LDL-C $<1.80 \mathrm{mmol} / \mathrm{L}$ did not (estimated change in means: $-1.4,95 \% \mathrm{CI}:[-5.9,3.0])$. We did not test any significant differences in the discharge NIHSS scores, length of hospital stays, discharge mRS scores or the in-hospital mortality (Table 3). Comparing with patients having LDL-C $\geq 1.80 \mathrm{mmol} / \mathrm{L}$, patients with LDL-C $<1.80$ $\mathrm{mmol} / \mathrm{L}$ had 0.84 odds to have favorable outcomes (OR: $0.84,95 \mathrm{CI}$ : $[0.49,1.45], \mathrm{P}=0.54)$, and 1,20 odds to dead (OR: $1.20,95 \% \mathrm{CI}$ : [0.39, $3.69], \mathrm{P}=0.75)$ at discharge.

Table 3: Outcomes of the indexed intracerebral hemorrhagic events

\begin{tabular}{|c|c|c|c|c|c|}
\hline & $\begin{array}{c}\text { LDL-C < } \\
1.80 \mathrm{mmol} / \mathrm{L} \\
(\mathrm{n}=31)\end{array}$ & $\begin{array}{c}\text { LDL-C } \geq \\
1.80 \mathrm{mmol} / \mathrm{L} \\
(\mathrm{n}=166)\end{array}$ & $\begin{array}{l}\text { All patients } \\
(n=197)\end{array}$ & Statistics & $P$ \\
\hline Discharge NIHSS scores, median (IQR) & $5(2-12)$ & $6(3-12)$ & $6(3-12)$ & $<0.001^{*}$ & 0.96 \\
\hline LOS, d, median (IQR) & $17(14-28)$ & $19(11-24)$ & $18(12-25)$ & $0.39^{*}$ & 0.53 \\
\hline Discharge mRS scores, median (IQR) & $3(4)$ & $3(2-5)$ & $3(2-5)$ & $0.11^{*}$ & 0.75 \\
\hline Discharge mRS scores $0-3, n,(\%)$ & $17(55 \%)$ & $98(59 \%)$ & $115(58 \%)$ & $0.19^{\dagger}$ & 0.66 \\
\hline In-hospital death, $\mathrm{n},(\%)$ & $2(6 \%)$ & $9(5 \%)$ & $11(6 \%)$ & 0.05 & 0.82 \\
\hline
\end{tabular}

${ }^{*}$ Kruskal-Wallis test; ${ }^{\dagger}$ Chi-Square test; ${ }^{*}$ Fisher's Exact test.

Abbreviations: NIHSS: National Institutes of Health Stroke Scale; IQR: interquartile range; LOS: length of Stay; mRS: modified Rankin Scale; LDLC: low-density lipoprotein cholesterol.

Figure 1: Factors predicting favorable outcomes of ICH.

\section{Odds Ratio $(95 \% \mathrm{Cl})$}

\begin{tabular}{|c|c|}
\hline Age & $1.00(0.97-1.03)$ \\
\hline Sex (Female vs. Male) & $1.21(0.58-2.55)$ \\
\hline ICH Score & $0.23(0.15-0.36)$ \\
\hline Blood Glucose & $1.00(0.97-1.03)$ \\
\hline $\begin{array}{l}\mathrm{LDL}-\mathrm{C}<1.8 \text { vs } . \geq 1.8 \\
\mathrm{mmol} / \mathrm{L}\end{array}$ & $1.40(0.48-4.01)$ \\
\hline
\end{tabular}

\begin{tabular}{lllllll}
\hline 0.00 & 100 & 2.00 & 3.00 & 4.00 & 5.00 & 6.00
\end{tabular}

Figure Legend: In the multiple logistic regression model, only baseline ICH scores were significantly associated with favorable outcomes (OR: 0.23, 95\% CI: [0.15-0.36]). Age, sex, baseline blood glucose or low-density lipoprotein cholesterol were not associated with outcomes. 


\section{Discussion}

ILLT, targeting an LDL-C $<1.80 \mathrm{mmol} / \mathrm{L}$ was not associated with the baseline neurological deficits of an $\mathrm{ICH}$. The outcomes at discharge were generally consistent, except that patients with LDL-C $<1.80 \mathrm{mmol} / \mathrm{L}$ failed to have significant improvements in the NIHSS scores at discharge than admission. An LDL-C $<1.80 \mathrm{mmol} / \mathrm{L}$ was associated with higher risks of secondary intraventricular hemorrhage.

Our outcomes were supported by previous studies. A retrospective study, which analyzed a total of 732 patients with acute ischemic stroke within 72 hours of symptoms onset, indicated that with each $1 \mathrm{mmol} / \mathrm{L}$ reduction in LDL-C levels, the risk of an unfavorable outcome (an mRS score of 36 points) was increased by $46.2 \%(\mathrm{OR}=0.538,95 \% \mathrm{CI}$ : [0.300, 0.964]; $\mathrm{P}=0.037$ ) in the patients with hemorrhagic transformation.[14] But the generality of the study [14] was limited, with the potential selection bias with the high hemorrhagic transformation rate (14.2\%). The Helsinki ICH Study was a single-center observational registry of consecutive $964 \mathrm{ICH}$ patients,[15] showing significantly lower LDL-C levels in patients who died at hospital than the survivors $(1.9$ vs. $2.4 \mathrm{mmol} / \mathrm{L} ; \mathrm{P}<0.001)$. Another multi-center stroke registry study from Taiwan indicated that patients with total cholesterol (TC) $<4.14 \mathrm{mmol} / \mathrm{L}$ presented more frequently with severe neurological deficits (NIHSS scores $\geq 15$ ) (adjusted OR:1.80); and 3-month unfavorable outcomes (mRS scores > 2) (adjusted OR: 1.41) when compared with the patients with $\mathrm{TC}>4.14$ mmol/L.[16] The retrospective cohort study from another stroke center in China found the rates of unfavorable outcomes $(\mathrm{mRS}>2)$ was higher in the ICH patients with lower LDL-C mmol/L (LDL-C $<1.80 \mathrm{mmol}$ vs. $2.59 \mathrm{mmol}$ vs. $>2.59 \mathrm{mmol} / \mathrm{L}: 57 \%$ vs $39 \%$ vs. $37 \%$; $\mathrm{P}<0.01)$.[17]

Other lipids was repoted to be associated with the incidence of ICH. Low plasma high-density lipoprotein cholesteral (HDL-C) $(<1.38 \mathrm{mmol} / \mathrm{l})$ may be associated with higher risks of the incidence of ICH.[18] The conclusion was consistance with the recent study: after a mean follow-up of 10.7 years, high HDL-C concentration was associated with a decreased incidence of ICH in women (HR: 0.23 ; 95\% CI: [0.06, 0.89]), but not in men (HR: 0.73; 95\% CI: [0.27, 1.97]).[19] Low serum triglyceride levels were associated with an increased risk of ICH (HR for highest versus lowest quartile: 0.20 95\% CI: [0.06, 0.69]).[20] Non-HDL-C were associated with increased risk of hemorrahatic transformation of ischemic stroke; compared with the highest quartiles, the first, second and third quartiles were associated with increased risk of HT (adjusted OR: 1.74; 95\% CI: [1.09, 2.78], 2.01, 95\% CI: [1.26, 3.20], and 1.76, 95\% CI: [1.10, 2.83], respectively, $P$ for trend $=0.024$ ). [21] With the current studies, the associations between LDL and the incidence of ICH was warrant to be tested.

There were many limitations within the current study. Firstly, the sample size was insufficient. In order to test the potential effects of the ILLT in the secondary prevention settings for ischemic stroke, we selected the patients with acute ICH as well as the medical histories of ischemic stroke as the study population. Secondly, the data was collected retrospectively from a single center, the knowledge achieved from the study was warranted to be testified by multiple centers. Thirdly, due to the limitations of the database, we did not adjust the blood pressure status at hospital, which may interfere the outcomes of the hemorrhagic events.

\section{Conclusion:}

ILLT achieved LDL-C $<1.80 \mathrm{mmol} / \mathrm{L}$ was associated with limited improvements in the neurological deficits in the patients with $\mathrm{ICH}$.

Acknowledgements:

The study was supported by the National Natural Science Foundation of China [grant numbers 82001242].

Acknowledgements: none.

Declarations of interest: none

\section{Reference}

1. Kernan W.N., Ovbiagele B., Black H.R., Bravata D.M., Chimowitz M.I., Ezekowitz M.D., Fang M.C., et al., Guidelines for the prevention of stroke in patients with stroke and transient ischemic attack: a guideline for healthcare professionals from the American Heart Association/American Stroke Association. Stroke, 2014. 45(7): 2160-2236.

2. Zhang Q., Liu S., Liu Y., Hua Y., Song H., Ren Y., Song Y., et al., Achieving low density lipoprotein-cholesterol<70mg/dL may be associated with a trend of reduced progression of carotid artery atherosclerosis in ischemic stroke patients. J Neurol Sci, 2017. 378: 26-29.

3. Hemphill J.C., Greenberg S.M., Anderson C.S., Becker K., Bendok B.R., Cushman M., Fung G.L., et al., Guidelines for the Management of Spontaneous Intracerebral Hemorrhage. Stroke, 2015. 46(7): 2032-2060.

4. An S.J., Kim T.J., and Yoon B.-W., Epidemiology, Risk Factors, and Clinical Features of Intracerebral Hemorrhage: An Update. Journal of stroke, 2017. 19(1): 3-10.

5. Judge C., Ruttledge S., Costello M., Murphy R., Loughlin E., Alvarez-Iglesias A., Ferguson J., et al., Lipid Lowering Therapy, Low-Density Lipoprotein Level and Risk of Intracerebral Hemorrhage - A Meta-Analysis. J Stroke Cerebrovasc Dis, 2019. 28(6): 1703-1709.

6. Amarenco P., Kim J.S., Labreuche J., Charles H., Giroud M., Lee B.C., Mahagne M.H., et al., Benefit of Targeting a LDL (Low-Density Lipoprotein) Cholesterol $<70 \mathrm{mg} / \mathrm{dL}$ During 5 Years After Ischemic Stroke. Stroke, 2020. 51(4): 1231-1239.

7. Ma C., Gurol M.E., Huang Z., Lichtenstein A.H., Wang X., Wang Y., Neumann S., et al., Low-density lipoprotein cholesterol and risk of intracerebral hemorrhage: A prospective study. Neurology, 2019. 93(5): e445-e457.

8. Chang J.J., Katsanos A.H., Khorchid Y., Dillard K., Kerro A., Burgess L.G., Goyal N., et al., Higher low-density lipoprotein cholesterol levels are associated with decreased mortality in patients with intracerebral hemorrhage. Atherosclerosis, 2018. 269: 14-20.

9. Elkhatib T.H.M., Shehta N., and Bessar A.A., Hematoma Expansion Predictors: Laboratory and Radiological Risk Factors in Patients with Acute Intracerebral Hemorrhage: A Prospective Observational Study. J Stroke Cerebrovasc Dis, 2019. 28(8): 2177-2186.

10. Cai B., Peng L., Wang Z.B., Zhang M., and Peng B., Association between Serum Lipid and Hematoma Expansion after Spontaneous Intracerebral Hemorrhage in Chinese Patients. J Stroke Cerebrovasc Dis, 2020. 29(6): 104793. 
11. Chen Y.W., Li C.H., Yang C.D., Liu C.H., Chen C.H., Sheu J.J., Lin S.K., et al., Low cholesterol level associated with severity and outcome of spontaneous intracerebral hemorrhage: Results from Taiwan Stroke Registry. PLoS One, 2017. 12(4): $\mathrm{e} 0171379$.

12. Goldstein L.B., Bushnell C.D., Adams R.J., Appel L.J., Braun L.T., Chaturvedi S., Creager M.A., et al., Guidelines for the primary prevention of stroke: a guideline for healthcare professionals from the American Heart Association/American Stroke Association. Stroke, 2011. 42(2): 517-584.

13. Hemphill J.C., 3rd, Bonovich D.C., Besmertis L., Manley G.T., and Johnston S.C., The ICH score: a simple, reliable grading scale for intracerebral hemorrhage. Stroke, 2001. 32(4): 891897.

14. Lv G., Wang G.Q., Xia Z.X., Wang H.X., Liu N., Wei W., Huang Y.H., et al., Influences of blood lipids on the occurrence and prognosis of hemorrhagic transformation after acute cerebral infarction: a case-control study of 732 patients. Mil Med Res, 2019. 6(1): 2.

15. Mustanoja S., Strbian D., Putaala J., Meretoja A., Curtze S., Haapaniemi E., Sairanen T., et al., Association of prestroke statin use and lipid levels with outcome of intracerebral hemorrhage. Stroke, 2013. 44(8): 2330-2332.

16. Chen Y.W., Li C.H., Yang C.D., Liu C.H., Chen C.H., Sheu J.J., Lin S.K., et al., Low cholesterol level associated with severity and outcome of spontaneous intracerebral hemorrhage: Results from Taiwan Stroke Registry. PLoS One, 2017. 12(4): $\mathrm{e} 0171379$.

17. Xie Y.-Y., Liu S.-M., Zhang Q., Jia Y., and Ding J.-P., Associations between low-density lipoprotein cholesterol and haemorrhagic stroke. Journal of geriatric cardiology: JGC, 2021. 18(3): 204-209.

18. Wang X., Li S., Bai Y., Fan X., Sun K., Wang J., and Hui R., Inverse association of plasma level of high-density lipoprotein cholesterol with intracerebral hemorrhage. Journal of lipid research, 2011. 52(9): 1747-1754.

19. Watanabe J., Kakehi E., Kotani K., Kayaba K., Nakamura Y., and Ishikawa S., High-Density Lipoprotein Cholesterol and Risk of Stroke Subtypes: Jichi Medical School Cohort Study. Asia Pacific Journal of Public Health, 2020. 32(1): 27-34.

20. Wieberdink R.G., Poels M.M.F., Vernooij M.W., Koudstaal P.J., Hofman A., van der Lugt A., Breteler M.M.B., et al., Serum Lipid Levels and the Risk of Intracerebral Hemorrhage: The Rotterdam Study. Arteriosclerosis, Thrombosis, and Vascular Biology, 2011. 31(12): 2982-2989.

21. Wang Y., Song Q., Cheng Y., Wei C., Ye C., Liu J., Wu B., et al., Association between non-high-density lipoprotein cholesterol and haemorrhagic transformation in patients with acute ischaemic stroke. BMC neurology, 2020. 20(1): 47-47.
This work is licensed under Creative Commons Attribution 4.0 License

\section{To Submit Your Article Click Here: Submit Manuscript}

DOI: $10.31579 / 2578-8868 / 200$
Ready to submit your research? Choose Auctores and benefit from:

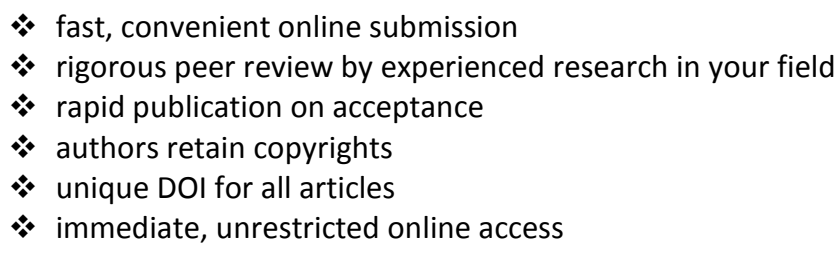

At Auctores, research is always in progress.

Learn more https://auctoresonline.org/journals/neuroscience-andneurological-surgery 